\title{
REFLEXIVE ALGEBRAS and SIGMA ALGEBRAS
}

\section{T.C. PRZYMUSINSKI and V.K. SRINIVASAN}

\author{
Department of Mathematics \\ University of Texas at E1 Paso \\ E1 Paso, Texas 79968 U.S.A.
}

(Received June 30, 1985)

\begin{abstract}
The concept of a reflexive algebra ( $\sigma$-algebra) $B$ of subsets of a set $X$ is defined in this paper. Various characterizations are given for an algebra ( $\sigma-a l g e b r a) \beta$ to be reflexive. If $\mathrm{V}$ is a real vector lattice of functions on a set $\mathrm{X}$ which is closed for pointwise limits of functions and if $\beta=\left\{A \mid A \subseteq X\right.$ and $\left.C_{A}(x) \varepsilon V\right\}$ is the $\sigma$-algebra induced by $V$ then necessary and sufficient conditions are given for $\beta$ to be reflexive (where $C_{A}(x)$ is the indicator function).
\end{abstract}

KEY WORDS AND PHRASES. Reflexive algebra, $\sigma$-algebra, and Boolean algebra. 1980 AMS SUBJECT CLASSIFICAIION CODES. 16 A30.

1. INTRODUCTION.

The object of this paper is to study the concept of reflexive algebra and a reflexive $\sigma$-algebra $\beta$ of subsets of a set $X$. The concept naturally arises, when we consider the topology generated by an algebra or $\sigma$-algebra $\beta$ on $X$. An algebra $\beta$ of subsets of $X$ is said to be reflexive if $\beta(\tau(\beta))=\beta$, where $\tau(\beta)$ is the topology generated on $X$ by taking $\beta$ as a base and $\beta(\tau)$ is the family of closed and open subsets of $X$ under a zero-dimensional topology $\tau$.

In section 2, we discuss some preliminaries concerning representations of algebras and we introduce some definitions. In section 3, various characterizations are given for an algebra to be reflexive. For a $\sigma$-algebra it is shown that an equivalent condition for it to be reflexive is that its real measurable functions should coincide with $\tau(\beta)$-real continuous functions. Thus for reflexive $\sigma$-algebras the study of real measurable functions amounts to the study of real continuous functions with respect to topology $\tau(\beta)$. Given any algebra $\beta$ there is the smallest reflexive algebra generated by $1 t$. An example is given to show that not every measure on a $\sigma$-algebra $\beta$ can be extended to the smallest reflexive o-algebra containing it.

If $V$ is a real vector lattice of functions on a set $X$ which is closed for pointwise limits of functions and if $B=\left\{A \mid A \subseteq X\right.$ such that $\left.C_{A}(x) \in V\right\}$ is the $\sigma-a l g e b r a$ induced by $V$, necessary and sufficient conditions are given for $\beta$ to be reflexive. 2. PRELIMINARIES AND DEFINITIONS.

Let $(X, \beta)$ be an arbitrary algebra of subsets of $X$. Define for $x, y$ in $X, X \sim y$ if for every $B \in B$, if $x \in B$ we have $y \in B$. It is easily seen that $\sim$ is an 
equivalence relation and the map $q: X \rightarrow X / \sim$ gives an algebra

$$
q(B)=\{q(B): B \in B\}
$$

in $X / \sim$ which is isomorphic to $\beta$. Moreover $q(\beta)$ is point-separating. In view of tha above procedure, we will in the sequel assume that all our algebras are point-separating.

Let $\beta^{*}$ denote any Boolean algebra which is isomorphic to $B$. Let $S(B)=S\left(B^{*}\right)$ denote the Stone-space of the Boolean algebra $\beta^{*}$. We note that $S(B)=$ $\left\{\lambda: \lambda\right.$ is a maximal filter in $\left.B^{*}\right\}$. On $S(B)$ the topology is generated by sets of the form $[B]=\left[B^{*}\right]=\left\{\lambda \in S(B): B^{*} \in \lambda\right.$ where $\left.B \in B\right\}$ where $B \rightarrow B^{*}$ is the isomorphism between $\beta$ and $\beta^{*}$. It is known that this topology $(S(\beta), \sigma)$ is a compact zerodimensional space and that the Boolean algebra of Clopen (closed and open) subsets of $S(B)$ is isomorphic to $\beta^{*}$ and thus isomorphic to $B$.

If $\Delta$ is any Boolean algebra and $(X, B)$ is such that $\Delta$ is isomorphic to $\beta$ then we say that $(X, B)$ is a representation of $\Delta$.

For each representation $(X, B)$ of a Boolean algebra $\beta^{*}$ there is a natural embedding

$$
\Psi:(X, \tau(\beta)) \rightarrow(S(B): \sigma)
$$

where $\tau(\beta)$ is the topology generated by $\beta$ on $X$, defined by

$$
\Psi(X)=\left\{B^{*} \in B^{*}: X \in B\right\}
$$

Then $\Psi(X)$ is a dense subspace of $S(B)$. Conversely, if $T$ is any dense subspace of $S(B)$ then $(T, \Delta)$ is a representation of $B^{*}$ where $\Delta=\{T \cap[B]: B \in B\}$. DEFINITION 1. A topological space $(X, \tau)$ is called a P-space if every $F_{\sigma}$ set in $X$ is closed.

3. MAIN RESULTS.

We start this section by first observing that for every $\beta$ the space $(X, \tau(\beta))$ is a zero-dimensional Hausdorff space. If further $\beta$ is a $\sigma$-algebra then $(X, \tau(\beta))$ is a P-space. However it can happen that $(X, \tau(\beta))$ may be a P-space without $\beta$ being a o-algebra as the ensuing simple example shows.

EXAMPLE 1. Let $\omega$ denote the first infinite cardinal and let

$$
B=\{A \subset \omega:|A|<\omega \text { or }|\omega-A|<\omega\} \text {. }
$$

Then $(X, \tau(B))$ is discrete and thus a P-space, while clearly $B$ is not a $\sigma$-algebra. ( $|A|$ is the cardinality of $A$ ).

Let $\tau$ denote a zero-dimensional topology on a set $x$. By defining $x$ y $(\mathrm{x}, \mathrm{y} \in \mathrm{X})$ if and only if for each $U \in \tau$ if $\mathrm{x} \in U$ we have $\mathrm{y} \in \mathrm{U}$, we obtain an equivlence relation. The quotient space $X / \sim$ is Hausdorff and zero-dimensional. In view of this, without loss of generality we will assume in the sequel that $(X, \tau)$ is itself a Hausdorff and zero-dimensional, and hence completely regular. We then denote by $\beta(\tau)$ the family of clopen subsets of $(X, \tau)$. We now have THEOREM 1. The family $\beta(\tau)$ is always an algebra on $X$. Moreover $B(\tau)$ is a $\sigma-a$ lgebra if and only if $(X, \tau)$ is a $P$-space.

PROOF. The first part is obvious. If $(X, \tau)$ is a P-space then the union of countably many clopen sets is clopen, which shows that $B(\tau)$ is a $\sigma$-algebra. Conversely, if the union of countably many clopen sets is clopen, $\tau$ is obviously a P-space.

The following facts are easily established:

$$
\begin{aligned}
& \tau(\beta(\tau))=\tau . \\
& \beta(\tau(\beta)) \supset \beta .
\end{aligned}
$$


In view of Example 1, it is seen that the reverse inclusion in (3.2) does not always hold. This prompts the following definition:

DEFINITION 2. An algebra $\beta$ of subsets of a set $X$ is reflexive if

$$
\beta(\tau(\beta))=\beta \text {. }
$$

EXAMPLE 2. Let $\omega_{1}$ denote the first uncountable cardinal and let

$$
B=\left\{A \subset \omega_{1}:|A| \leq \omega \text { or }\left|\omega_{1}-A\right| \leq \omega\right\}
$$

Then $\beta$ is a non reflexive $\sigma$-algebra on $\omega_{1}$. In this case $\beta(\tau(\beta))=P\left(\omega_{1}\right)$, the set of all subsets of $\omega_{1}$. However if

$$
\begin{gathered}
\left.\bar{\omega}_{1}=\text { ordinals } \alpha: \quad \alpha \leq \omega_{1}\right\} \text { and further if } \\
B=\left\{\mathrm{A} \subset \bar{\omega}_{1} \quad \text { such that either }\left|\bar{\omega}_{1}-\mathrm{A}\right| \leq \omega \text { or }|\mathrm{A}| \leq \omega \text { and } \omega_{1} \notin \mathrm{A}\right\} .
\end{gathered}
$$

then $\beta$ is a non trivial reflexive $\sigma$-algebra on $\bar{\omega}_{1}$.

LEMMA 1. $\beta(\tau(\beta(\tau(\beta))))=\beta(\tau(\beta))$ and hence $\beta(\tau(\beta))$ is always reflexive.

PROOF. Since $\tau(B(\tau))=\tau$, it follows that

$$
B(\tau(\beta(\tau(\beta))))=B(\tau(\beta)) \text {. }
$$

LEMMA 2. For every algebra $\beta$, the algebra $R_{\beta}=\beta(\tau(\beta))$ is the smallest reflexive 'algebra that contains $\beta$. If further $\beta$ is a $\sigma$-algebra so is $R_{\beta}$.

PROOF. In view of $\beta(\tau(\beta)) \supset \beta$, it follows that $\beta \subset R_{\beta}$ and by Lemma 1 , $R_{B}$ is reflexive. If $\Omega$ is any reflexive algebra such that

$$
B \subset \Omega \subset R_{B}
$$

then $\Omega=\beta(\tau(\Omega))=\beta(\tau(\beta))=R_{B}$ and hence $R_{B}$ is minimal. If $R_{B}$ is a o-algebra, by an earlier result it follows that $\tau\left(R_{\beta}\right)$ is a P-space and hence $R_{\beta}=\beta\left(\tau\left(R_{\beta}\right)\right)$ is a o-algebra. This completes the proof.

We now note the following two properties:

$$
\begin{aligned}
& \text { Always } B \subset \tau(B) \text {. } \\
& \text { Always } B(\tau) \subset \tau \text {. }
\end{aligned}
$$

THEOREM 2. For a Boolean algebra $\beta$, the following conditions are equivalent:

$$
B=\tau(B) \text {. }
$$

(ii) $R$ is reflexive and for each $x \in X,\{x\} \in \beta$.

(iii) $\beta=P(X)$ i.e. $\beta$ is trivial.

PROOF. (i) $\Rightarrow$ (iii). By (i) each open set in $\tau(\beta)$ is closed and hence every point is open and thus $\tau(B)$ is discrete. Hence $B=P(X)$.

That $(\mathrm{iii}) \Rightarrow$ (ii) is obvious.

We now prove that $(i i) \Rightarrow(i)$. Since $\beta$ is reflexive, $\beta(\tau(\beta))=\beta$ and since all points belong to $\beta$, all one-point sets are open in $\tau(\beta)$. Thus $\tau(\beta)$ is discrete. Hence $B(\tau(B))=\beta=P(X)$ which implies (i).

THEOREM 3. If $\beta$ is a reflexive $\sigma$-algebra and if $(X, \tau(\beta))$ is such that all one-point subsets of $X$ are $G_{\delta}$ sets in $\tau(\beta)$ then $\beta=P(X)$.

PROOF. Since $\beta$ is a $\sigma$-algebra, $\tau(\beta)$ is a P-space and thus it must be discrete. But $\beta=\beta(\tau(\beta))$ and hence $\beta=P(X)$. 
THEOREM 4. For topology $\tau$ the following conditions are equivalent:

(i) $\tau=\beta(\tau)$.

(ii) $\tau$ is discrete.

(iii) $\tau=P(X)$.

PROOF. Since all open sets are clopen, $\tau$ is discrete and hence $\tau=P(X)$.

DEFINITION 3. A compact Hausdorff space $Z$ is called Banaschewski compactification of its dense subspace $X$, if for every clopen set $U$ in $X$,

$$
\bar{U}^{Z}(\overline{\mathrm{X}-\mathrm{U}})^{Z}=\phi \text {, where }
$$

$\bar{A} Z$ means the closure taken in $Z$.

THEOREM 5. For an algebra $\beta$ the following statements are equivalent:

(i) $B$ is reflexive .

(ii) $\quad \beta=B(\tau)$ for some topology $\tau$ on $X$.

(iii) $S(B)$ is the Banaschewski compactification of $(X, \tau(B))$.

(iv) If $C \subset X$ and $C=U B^{\prime}$ and $X-C=\cup B^{\prime \prime}$, where $B^{\prime}$ and $B^{\prime \prime}$ are subsets of $B$, then $C \in B$ (here $C=\cup \beta^{\prime}$ means that $C$ is union of sets from $\beta^{\prime}$ ).

PROOF. (i) $\Rightarrow$ (ii). Since $\beta=\beta(\tau(\beta))$, it is sufficient to take $\tau=\tau(\beta)$.

(ii) $=>$ (iv). If $C$ is as in (iv) and if $\tau$ is as in (ii) then $C$ is clopen in $\tau$ ' and thus $C \in B$.

(iv) $\Rightarrow$ (iii). Suppose $U$ is a clopen set in (X, $\tau(\beta)$ ) then by (iv) $U \in \beta$. Hence $\bar{U} \cap(\overline{X-U})=\phi$, where closure is taken in $S(B)$.

(iii) $\Rightarrow$ (i) Suppose that $U$ is clopen in $\tau(B)$ then $\bar{U} \cap(\overline{X-U})=\phi$ in $S(B)$ and thus $U=[B] \cap X$, for some $B \in \beta$ which implies that $U \epsilon \beta$.

This completes the proof.

THEOREM 6. For a $\sigma$-algebra $\beta$ the following statements are equivalent:

(i) $B$ is reflexive.

(ii) $\quad \beta=B(\tau)$ for some $P$-topology $\tau$.

(iii) $S(B)$ is the Stone-C̃ech compactification of $(X, \tau(\beta))$.

(iv) The $(X, B)$-real measurable functions coincide with $(X, \tau(B))$-real continuous functions.

PROOF. (i) $\Rightarrow$ (ii). It suffices to take $\tau=\tau(\beta)$.

(ii) $\Rightarrow($ iv). Clearly $(X, \beta)$-measurability implies (X, $\tau(\beta))$-continuity.

Conversely if $f: X \rightarrow R$ is continuous, then the inverse images of open sets in $R$ are open $F_{\sigma}$-sets in $(X, \tau(B))$ and these are clopen, since $\tau(B)$ is a P-space. Thus inverse images of open sets belong to $\beta(\tau(\beta))$. As $\beta=\beta(\tau)$ it follows that $\beta(\tau(\beta))=\beta$ and thus $f$ is measurable.

(iv) $\Rightarrow$ (iii). Let $f: X \rightarrow R$ be $\tau(\beta)$-continuous. Thus $f$ is $(X, \beta)-$ measurable and hence there exists a $B \in B$ such that $f^{-1}(0)^{C} \quad[B] . f^{-1}(1) \cap[B]=\phi$ and thus

$$
{\overline{\mathrm{f}^{-1}(0)}}^{\mathrm{S}(\beta)}{\frac{\mathrm{f}}{\mathrm{f}^{-1}(1)}}^{\mathrm{S}(\beta)}=\phi .
$$

This proves that (iv) implies (iii).

(iii) $\Rightarrow$ (i). The proof of this implication is the same as in Theorem 5 . 
THEOREM 7. For a Boolean algebra $\beta^{*}$ the following statements are equivalent:

(i) $\beta^{*}$ is complete.

(ii) Every representation $(X, B)$ of $\beta^{*}$ is reflexive.

PROOF. (i) $\Rightarrow(i i)$. If $\beta^{*}$ is complete then $S\left(\beta^{*}\right)$ is extremally disconnected. Let $X \subset S\left(\beta^{*}\right)$ be a dense subspace of $S\left(B^{*}\right)$ and let

$$
B=\left\{[B] \cap X: B \in B^{*}\right\}
$$

Suppose that $U \subset X$ is clopen in $X$. Then there exist disjoint open sets $U^{*}$ and $V^{\star}$ in $\mathrm{S}(B)$ with $\mathrm{U}^{\star} \cap \mathrm{X}=\mathrm{U}$ and $\mathrm{V}^{\star} \cap \mathrm{X}=\mathrm{X}-\mathrm{U}$. Then $\overline{\mathrm{U}^{\star}} \cap \overline{\mathrm{V}^{\star}}=\phi$, and hence $\overline{\mathrm{U}^{*}}$ is clopen. This means that $\overline{U^{\star}} \in \beta^{\star}$ and $\overline{U^{\star}} \cap \mathrm{X}=\mathrm{U} \in \beta$. This proves that (i) $\Rightarrow$ (ii). Conversely, suppose $B^{\star}$ is not complete. Then there exist open sets $U$ and $V$ in $S\left(\beta^{*}\right)$ such that $U=\operatorname{Int}(\bar{U})$ and $\bar{U} \cap \bar{V} \neq \phi$, but $U \cap V=\phi$. Let $X=(U \cap V, \sigma)$. Then $X$ is dense in $S\left(\beta^{*}\right)$ and $U$ is clopen in $X$ but $U \notin 3$. Hence $B$ is not reflexive. This completes the proof.

One of the relevant questions is that whether a measure defined on $\Sigma$ can be extended to the smallest reflexive $\sigma$-algebra $\beta(\tau(\beta))$ containing $\beta$. The following easy example shows that this may not be always possible.

EXAMPLE 3. If $X$ is a set of cardinality $2^{c}$, let

$$
\begin{aligned}
& B=\{B \subset X:|B| \leq \omega \text { or }|X-B| \leq \omega\} \text { and } \\
& \mu(B)=\left\{\begin{array}{l}
0, \text { if } B \text { is countable } \\
1, \text { otherwise }
\end{array}\right.
\end{aligned}
$$

Then $\beta$ is a $\sigma$-algebra and $\mu$ is a two valued measure on $B$. Clearly $B(\tau(\beta))=P(X)$. Since $2^{\mathrm{c}}$ is not measurable $\mu$ does not have an extention.

In the next theorem the following question is discussed. Let $V$ be a vector lattice of real funcitons on a set $X$ which is closed under pointwise limits of functions in $V$. If $C_{A}(x)$ is the indicator function of the subset $A \subseteq X$, then it is known that the collection

$$
B=\left\{A \subseteq X: \quad C_{A} \in V\right\}
$$

is a $\sigma$-algebra and that $\mathrm{V}$ is precisely the set of real $\beta$-measurable functions. The next theorem gives a characterization for $\beta$ to be reflexive.

THEOREM 8. Let $V$ be a vector lattice of real functions defined on a set $X$ and let $\mathrm{V}$ be closed under pointwise limits. Let

$$
B=\left\{A \subseteq X: C_{A} \in V\right\}
$$

Then $B$ is reflexive if and only if for each $f: X \rightarrow R$ such that $f=\operatorname{Sup}_{\alpha}\left\{g_{\alpha}\right\}=$ $\operatorname{Inf}_{\beta}\left\{h_{\beta}\right\}$, where $g_{\alpha} \in V, h_{B} \in V$, we have $f \in V$.

PROOF. In this result we use (iv) of Theorem 5. Suppose $A=U B^{\prime}$ and $X-A=U B^{\prime \prime}$, where $B^{\prime}, B^{\prime \prime} \subset B$. Let $g_{B}=C_{B}(x)$ and $h_{B}=C_{X-B}(x)$. Then clearly

$$
\operatorname{Sup}_{B \in B^{\prime}}\left\{g_{B}\right\}=C_{A}(x)=\operatorname{Inf}_{B \in B^{\prime \prime}}\left\{h_{B}\right\}
$$

Hence $C_{A} \in V$ and thus $C \in B$ which shows that $B$ is reflexive. 
Conversely, if $\beta$ is reflexive then $\tau(\beta)$-continuous functions are measurable. Thus a function $f$ which is both upper semi-continuous and lower semi-continuous is continuous and hence it is measurable. Thus $f \in V$.

The proof is complete.

\section{REFERENCES}

1. АВвот, J. C., Sets, lattices and Boolean Algebras, Allyn and Bacon, 1969.

2. HALMOS, P. R., Lectures on Boolean Algebras, Springer Verlag, New York, 1974.

3. SIKORSKI, R. Boolean Algebras, Ergebnisse der Mathematik und Ihrer Grenzgebiete, New Series, Vol. 25, Springer Verlag, New York, 1964. 


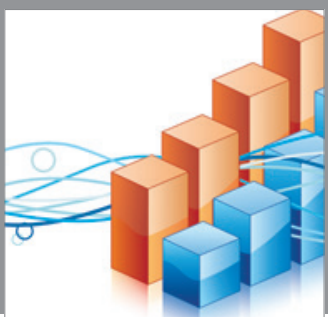

Advances in

Operations Research

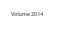

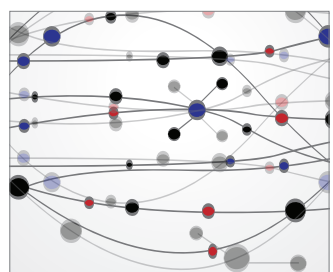

\section{The Scientific} World Journal
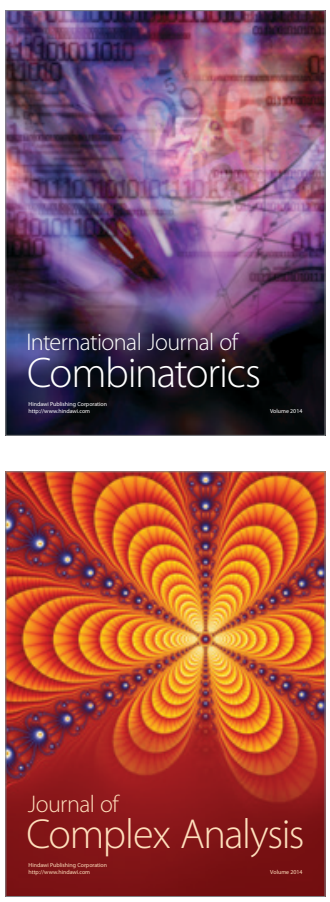

International Journal of

Mathematics and

Mathematical

Sciences
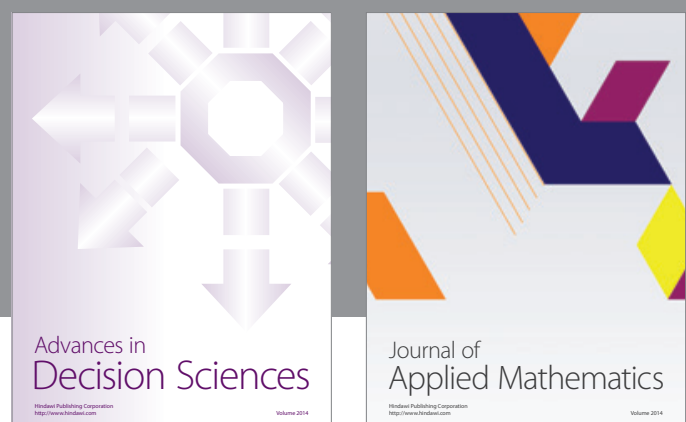

Journal of

Applied Mathematics
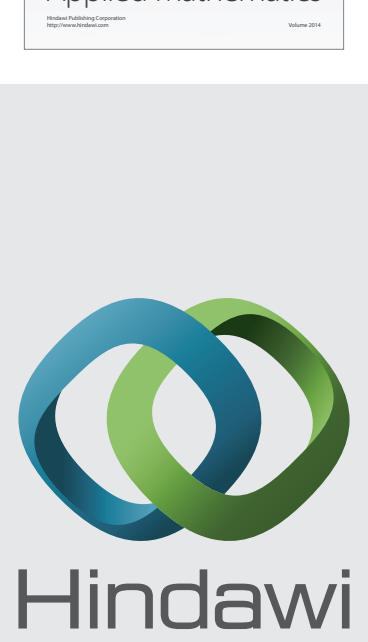

Submit your manuscripts at http://www.hindawi.com
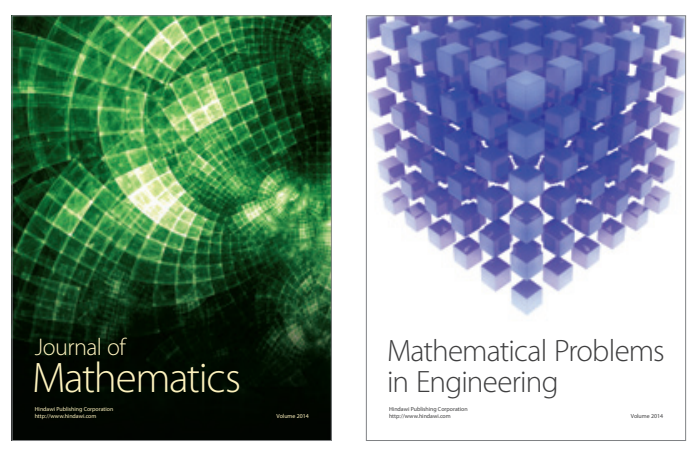

Mathematical Problems in Engineering
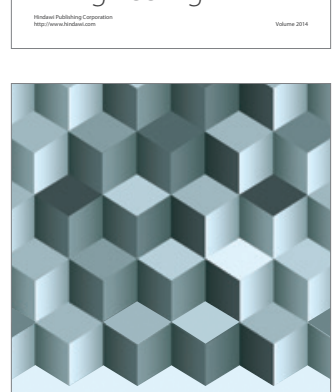

Journal of

Function Spaces
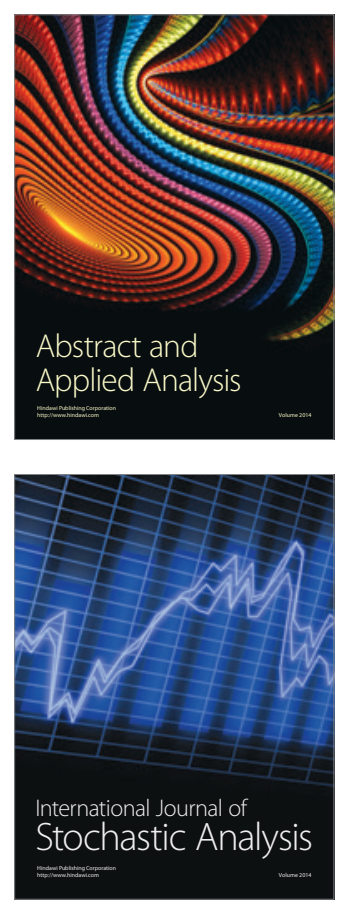

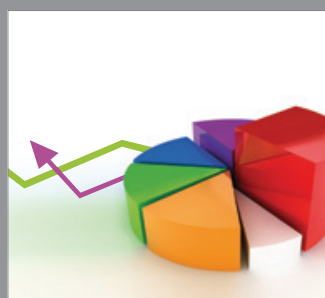

ournal of

Probability and Statistics

Promensencen
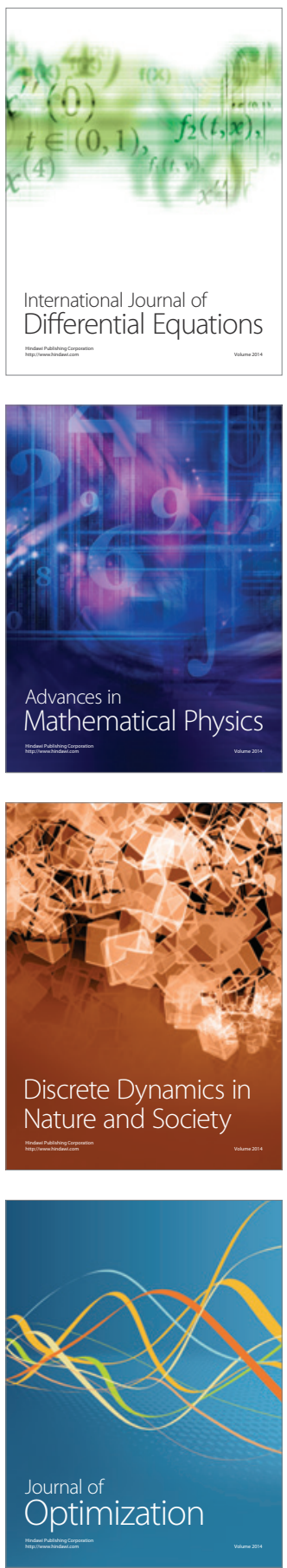